\title{
Effects of Dietary Supplementation with n-3 Fatty Acids Compared with n-6 Fatty Acids on Bronchial Asthma
}

\author{
Makoto Okamoto, Fumihiro Mitsunobu, Kozo Ashida, Takashi Mifune, Yasuhiro Hosaki, Hirofumi Tsugeno, \\ Seishi HaRada and Yoshiro TANIZAKI
}

\begin{abstract}
Objective The effects of perilla seed oil ( $n-3$ fatty acids) on bronchial asthma were compared with the effects of corn oil ( $n-6$ fatty acids) in relation to the pulmonary function and the generation of leukotriene B4 (LTB4) and C4 (LTC4) by leucocytes.

Methods and Subjects 14 asthmatic subjects were divided randomly into two groups: one group ( 7 subjects) consumed perilla seed oil-rich supplementation and the other group ( 7 subjects) consumed corn oil-rich supplementation for 4 weeks. Generation of LTs by leucocytes and respiratory function were compared between the two groups.

Results The generation of LTB4 and LTC4 by leucocytes tended to increase in subjects $(\mathrm{N}=7)$ with corn oil-rich supplementation, and decrease in subjects $(\mathrm{N}=7)$ with perilla seed oil-rich supplementation. Significant differences between the two groups were observed in the generation of LTB4 at 2 weeks $(p<0.05)$ and LTC4 at 2 weeks $(p<0.05)$ after dietary supplementation. Significant increases in the value of PEF $(p<0.05)$, FVC $(p<0.01)$, FEV $_{1.0}(p<0.05)$ and $\dot{\mathrm{V}}_{25}(\mathbf{p}<0.05)$ were found in subjects who received perilla seed oil supplementation for 4 weeks. And significant differences in the value of FVC $(p<0.05)$ and $\operatorname{FEV}_{1.0}(p<0.05)$ were observed between the two groups after 4 weeks of dietary supplementation.

Conclusion These results suggest that perilla seed oilrich supplementation is useful for the treatment of asthma in terms of suppression of LTB4 and LTC4 generation by leucocytes, and improvement of pulmonary function. (Internal Medicine 39: 107-111, 2000)
\end{abstract}

Key words: perilla seed oil, $\alpha$-linolenic acid, leukotriene B4, leukotriene $\mathrm{C} 4$, respiratory function

\section{Introduction}

Bronchial allergen challenge induces an immediate asthmatic reaction (IAR) within 30 minutes and a late asthmatic reaction (LAR), which occurs 6-8 hours after the challenge. The LAR, in which inflammatory cells such as lymphocytes, neutrophils, eosinophils and basophils migrate into allergic reaction sites in the airway $(1,2)$, is closely associated with bronchial hyperresponsiveness $(3,4)$. Leukotrienes (LTs) are among the major chemical mediators in asthma, particularly in the LAR, and are synthesized by inflammatory cells in large amounts during allergic reactions.

LTs are generated from arachidonic acid (AA), which is released from membrane phospholipids during cell activation, through the 5-lipoxygenase pathway (5). LTB4 and LTC4 are generated from linoleic acid (LA) through AA, and LTB5 from $\alpha$-linolenic acid ( $\alpha$-LNA) through eicosapentaenoic acid (EPA) through the same 5-lipoxygenase pathway. However, the action of LTB5 is much weaker than that of LTB4.

Dietary supplementation with perilla seed oil, which is rich in $\alpha$-LNA, has been expected to suppress the LT generation by leucocytes and increase the generation of LTB5. Conversely, supplementation with corn oil, which is rich in LA, is expected to increase the generation of LTB4 and LTC4, and decrease the generation of LTB5 by leucocytes. In a previous study (6), we showed the inhibitory effects of a diet containing perilla seed oil, a vegetable oil rich in $\alpha$-LNA, on the generation of LTs by leucocytes.

In this study, we examined the effects of perilla seed oilrich supplementation versus corn oil-rich supplementation in patients with bronchial asthma.

\section{Subjects and Methods}

The subjects of this study were 14 patients ( 6 men and 8 women) who were admitted to our hospital for the treatment of asthma. Seven subjects were atopic and the others were nonatopic. All subjects had moderately severe type asthma. The

From the Department of Medicine, Misasa Medical Branch, Okayama University Medical School, Tottori

Received for publication March 11, 1999; Accepted for publication August 23, 1999

Reprint requests should be addressed to Dr. Makoto Okamoto, the Department of Medicine, Misasa Medical Branch,Okayama University Medical School, Yamada 827, Misasa, Tohaku-gun, Tottori 682-0192 
mean duration of asthma was 15 years. All patients were treated with long-acting oral theophylline, inhaled $\beta_{2}$ adrenergic agonists and inhaled glucocorticosteroid (beclomethasone dipropionate: BDP) regularly. The mean dose of inhaled BDP was $196.4 \pm 173.7 \mu \mathrm{g} / \mathrm{day}$. Their mean age was 58.9 years (range, 22 to 84 years) and the mean of serum IgE was $1,003 \mathrm{IU} / \mathrm{ml}$ (range, 21 to $6,300 \mathrm{IU} / \mathrm{ml}$ ).

Bronchial asthma was evaluated according to the criteria of the International Consensus of Diagnosis and Management of Asthma (7). All patients had reversible airway response, as indicated by a $15 \%$ or a greater increase in their forced expiratory volume in one second $\left(\mathrm{FEV}_{1.0}\right)$ after inhaled bronchodilator use. The study was approved by the Institutional Human Investigation Committee at our hospital. Informed consent for the study protocol was obtained from all patients. Peak expiratory flow (PEF) in the early morning was recorded in all subjects using a peak flow meter (Assess: Health Scan Products Inc., Cedar Grove, NJ, USA).

The subjects were divided randomly into two groups: one group (7 subjects) consumed 10-20 grams of corn oil per day as salad dressing and/or mayonnaise instead of other oils for 4 weeks and the other group ( 7 subjects) consumed the same amount of perilla seed oil per day for 4 weeks. Other dietary components were not changed, and the amount of oil used in the diet and supplemented diet were recorded throughout the study period.

Pulmonary function tests, forced vital capacity (FVC), forced expiratory volume in one second $\left(\mathrm{FEV}_{1.0}\right)$ and $\dot{\mathrm{V}}_{25}$ were performed when patients were attack free using a Chestac 33 (Chest Co., Tokyo) linked to a computer.

The generation of LTB4 and LTC4 by peripheral leucocytes was assessed by a method previously described $(8,9)$. Cells were separated by counterflow centrifugation elutriation with a JE 6B rotor (Beckman Co., Geneva, Switzerland) (10), as described previously (11). The number of the cells was then adjusted to $5 \times 10^{6} / \mathrm{ml}$ in Tris ACM (composition: $1 \mathrm{ml}$ of 0.1 $\mathrm{mol} / / \mathrm{Ca}^{2+}, 0.5 \mathrm{ml}$ of $0.1 \mathrm{~mol} / / \mathrm{Mg}^{2+}$ and $98.5 \mathrm{ml}$ Tris A buffer; Trizma preset crystal, pH 7.7; Sigma Chemical Co., St. Louis, Mo, USA). The Ca ionophore A23187 $(1 \mu \mathrm{g})$ was added to the cell suspension. The mixed solution was incubated for $15 \mathrm{~min}-$ utes at $4^{\circ} \mathrm{C}$. Quantification of LTB 4 and LTC 4 by HPLC analysis was performed by a method described by Lam et al (12). The extraction of LTs was performed using a C18 Seppak (Waters Associates, Milford, MA). The concentrations of LTB4 and LTC4 were analyzed by HPLC system Model 510 (Waters Associates, Milford MA), equipped with an ultraviolet detector. The column used was a $5 \mathrm{~mm} \times 10 \mathrm{~cm}$ Radial-Pax cartridge (Shimazu Co., Kyoto). The results were expressed as $\mathrm{ng} / 5 \times 10^{6}$ cells.

The changes in LTB4, LTC4 and ventilatory parameters (PEF, FVC, $\mathrm{FEV}_{1.0}$ and $\dot{\mathrm{V}}_{25}$ ) values after dietary supplementation were expressed as the ratios of values before and after the supplementation.

The results were expressed as mean \pm standard deviation (SD). Statistically differences between means were estimated using the Student's t-test. A $p$ value of $<0.05$ was regarded as significant.

\section{Results}

The generation of LTB4 by leucocytes tended to increase in subjects with corn oil-rich supplementation, and to decrease in subjects with perilla seed oil-rich supplementation. The difference in the changes of LTB4 generation between the two groups was significant at two weeks after the supplementation $(\mathrm{p}<0.05)$, as shown in Fig. 1. The generation of LTC4 by leucocytes also

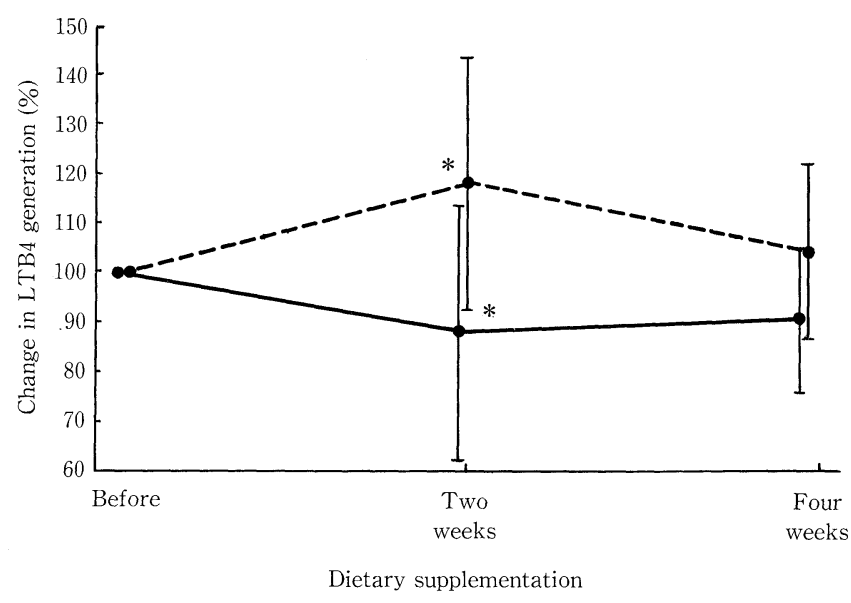

Figure 1. Changes of LTB4 generation by leucocytes after dietary supplementation for 4 weeks. A significant difference was observed between the two groups at two weeks after dietary supplementation. - - : perilla seed oil-rich supplementation group. $\cdots \cdots$ : corn oil-rich supplementation group $*$ : $\mathbf{p}<0.05$, LTB4: Leukotriene B4.

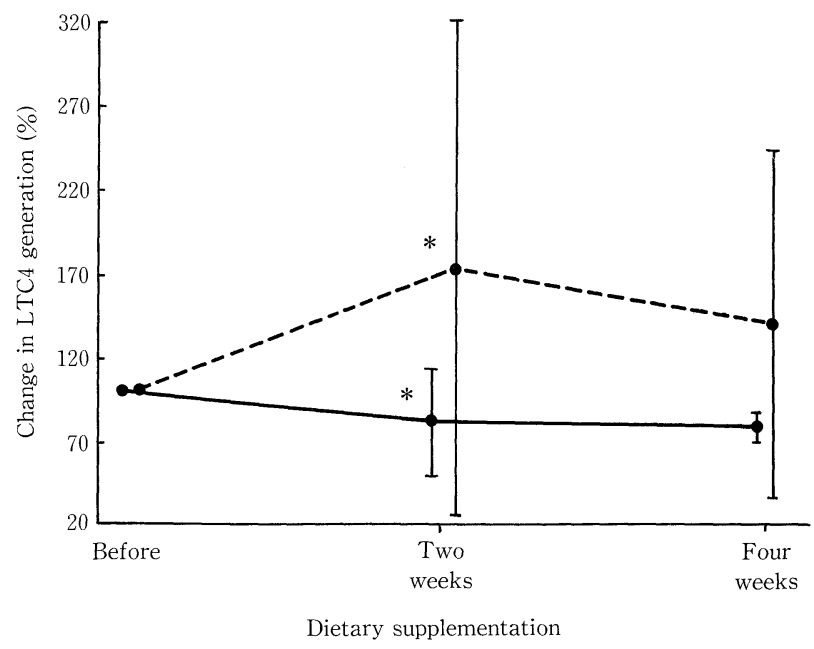

Figure 2. Changes of LTC4 generation by leucocytes after dietary supplementation for 4 weeks. A significant difference was observed between the two groups at two and four weeks after dietary supplementation. - - : perilla seed oil-rich supplementation group. $\cdots \cdots$ : corn oil-rich supplementation group *: p<0.05, LTC4: Leukotriene C4. 
increased in subjects with corn oil-rich supplementation. In contrast, LTC4 generation showed a tendency to decrease in subjects with perilla seed oil-rich supplementation, and significant differences in the LTC4 generation were observed between the two groups at 2 weeks after each supplementation $(\mathrm{p}<0.05)$ (Fig. 2).

The values of PEF in the morning increased in the two groups at 4 weeks after dietary supplementation. A significant increase in PEF was observed at 4 weeks after perilla seed oil-rich

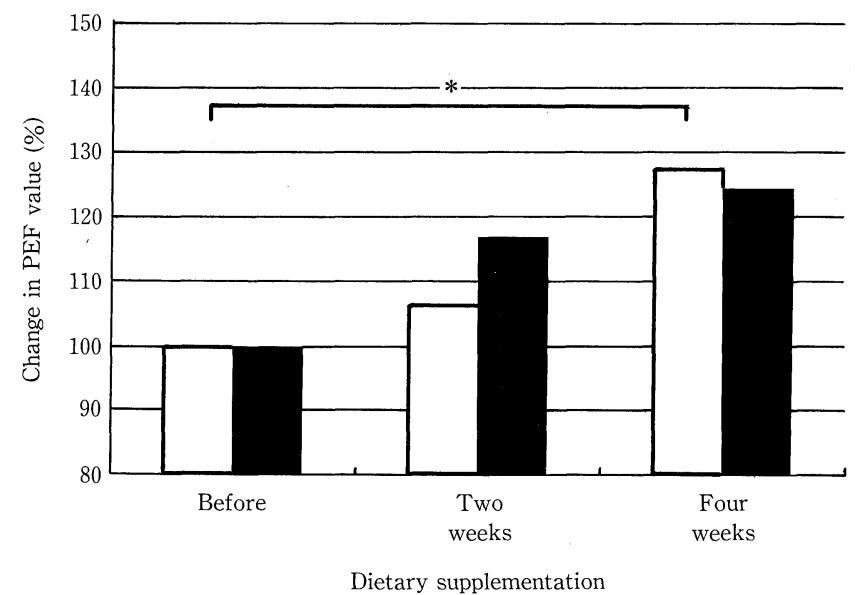

Figure 3. Changes of PEF in the morning after dietary supplementation for 4 weeks. A significant increase in PEF value was observed 4 weeks after perilla seed oil-rich supplementation. $\square$ : perilla seed oil-rich supplementation group. $\square$ : corn oil-rich supplementation group. *: p<0.05, PEF: peak expiratory flow (before inhalation of $\beta_{2}$ adrenergic agonists).

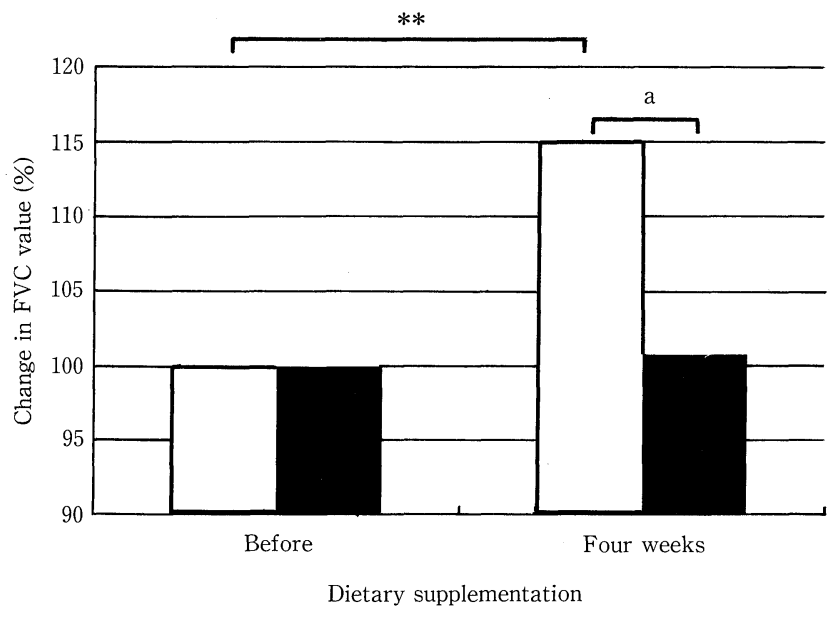

Figure 4. Changes of FVC in the two groups. A significant increase in the FVC value was observed at four weeks after perilla seed oil-rich supplementation. A significant difference was also observed between the two groups at 4 weeks after dietary supplementation. $\square$ : perilla seed oil-rich supplementation group.

: corn oil-rich supplementation group. ${ }^{* *}: \mathbf{p}<0.01$, a: $\mathbf{p}<0.05$, FVC: forced vital capacity. supplementation $(\mathrm{p}<0.05)$ (Fig. 3). A significant increase in the value of FVC was observed at 4 weeks after perilla seed oil-rich supplementation $(\mathrm{p}<0.01)$ and significant difference was observed between the two groups at 4 weeks after dietary supplementation $(\mathrm{p}<0.05)$ (Fig. 4). The $\mathrm{FEV}_{1.0}$ value was also significantly increased at 4 weeks after dietary supplementation with perilla seed oil and a significant difference was observed between the two groups at 4 weeks after dietary supplementation ( $\mathrm{p}<0.05$ ) (Fig. 5). The $\dot{\mathrm{V}}_{25}$ value was significantly

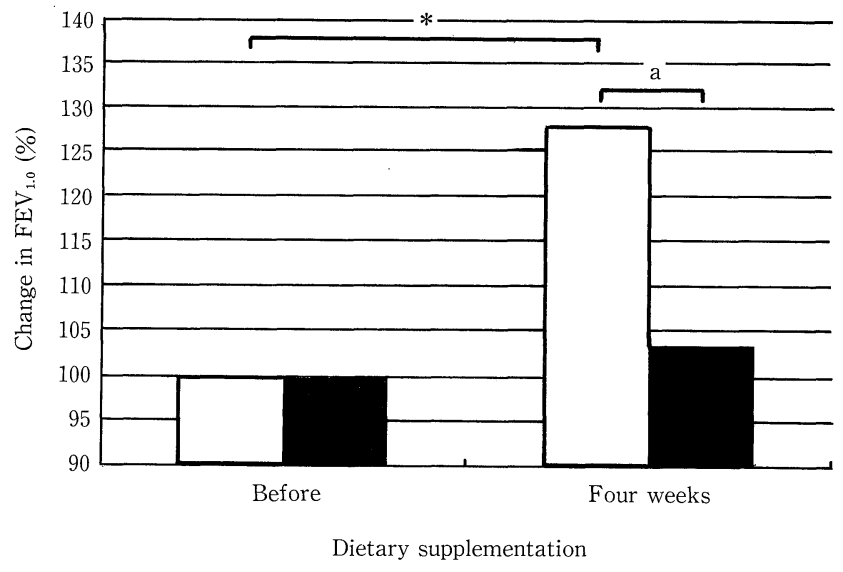

Figure 5. Changes of $\mathrm{FEV}_{1.0}$ value in the two groups after dietary supplementation. A significant increase in FEV $_{1.0}$ value was observed at 4 weeks after perilla seed oil-rich supplementation. A significant difference was also observed between the two groups at 4 weeks after dietary supplementation. $\square$ : perilla seed oil-rich supplementation group. $\square$ : corn oil-rich supplementation group. *: $\mathbf{p}<0.05$, a: $\mathbf{p}<0.05, \mathrm{FEV}_{1.0}$ : forced expiratory volume in one second.

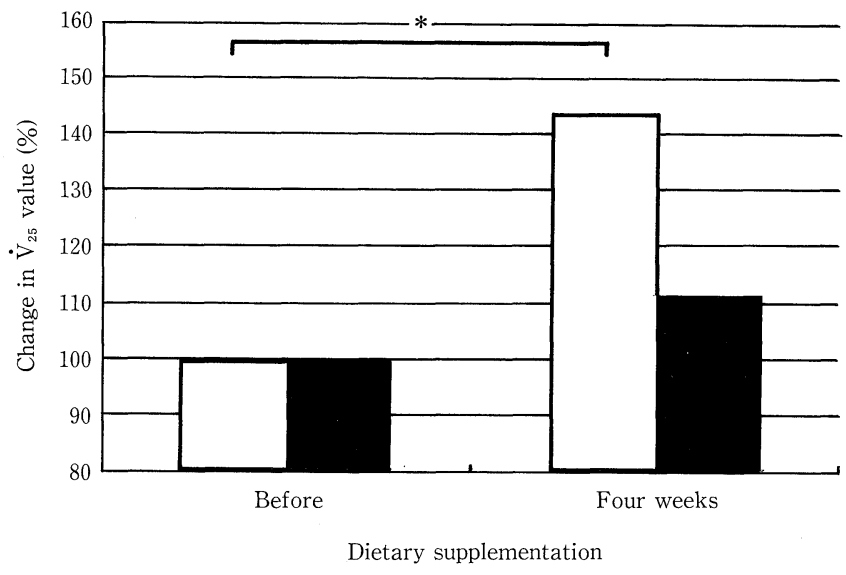

Figure 6. Changes of $\dot{\mathbf{V}}_{25}$ value in the two groups after dietary supplementation. Significant increase in $\mathbf{V}_{25}$ value was observed at 4 weeks after perilla seed oil-rich supplementation. $\square$ : perilla seed oil-rich supplementation group. $\square$ : corn oil-rich supplementation group. $*$ : $\mathbf{p}<\mathbf{0 . 0 5}$. 


\section{Окамото et al}

increased at 4 weeks after dietary supplementation with perilla seed oil $(\mathrm{p}<0.05)$ (Fig. 6). However, no significant increase in the values of these ventilatory parameters were found after corn oil-rich supplementation and no correlation between LTs and these ventilatory parameters was observed. Eosinophil numbers of the two groups did not change significantly during this study.

\section{Discussion}

Asthma is characterized by airway inflammation, bronchial hyper responsiveness to non-specific stimuli, and episodic and reversible airflow obstruction. Airway inflammation is the main patho-physiological manifestation of asthma. Leukotrienes (LTs) are among the most important chemical mediators released from inflammatory cells, which are involved in the pathogenesis of asthma. LTB4 acts by recruiting and activating inflammatory cells, particularly neutrophils, and promoting edema. The mediators play an important role in the asthmatic response by recruiting leukocytes to allergic reaction sites in the airway. The mediators have a bronchoconstrictor action and participate in the onset of asthma attacks $(1,9)$.

It has been reported that the generation of LTB4 is reduced by $n-3$ fatty acids $(13,14)$. LTB4 generated from LA and LTB5 generated from $\alpha$-LNA have similar biological activities. However, the action of LTB5 is much weaker than that of LTB4. The cysteinyl LTs (LTC4, LTD4 and LTE4) are implicated in the pathogenesis of allergen-induced airway responses as potent contractile agonists for airway smooth muscle and they mediate a later part of immediate airway obstruction; a fall in $\mathrm{FEV}_{1.0}$ after allergen exposure $(15,16)$.

Polyunsaturated fatty acids (PUFAS) of the $n-3$ series, EPA and docosahexaenoic acid (DHA), suppress the production of LTs by antagonistic metabolism, which occurs at the level of LT hydrolase through the 5-lipoxygenase pathway, and therefore they have the potential to alter LTs generation by leucocytes (17). These PUFAs have been reported to show anti-inflammatory effects in patients with chronic inflammatory diseases such as rheumatoid arthritis, psoriasis, and chronic inflammatory bowel disease (18-24). Several reports have focused on the beneficial effects of EPA or fish oil on bronchial asthma (25-29).

In the present study, the effects of $\alpha$-LNA rich perilla seed oil on asthma were examined in comparison with the effects of LA-enriched corn oil. The results revealed that $\alpha$-LNA-rich perilla seed oil supplementation suppressed the generation of LTB4 and LTC4 more strongly than LA-rich corn oil supplementation at two weeks of dietary supplementation. Although the exact reason for the disappearance of the perilla seed oil superiority is unknown, possible reasons are thought to be follows: the sample size was rather small to detect a true effect and increase in LA production in compensation for decreased levels of LTB4 and LTC4. Furthermore, the subjects given perilla seed oil-rich supplementation showed significantly higher increases of PEF, FVC, $\mathrm{FEV}_{1.0}$ and $\dot{\mathrm{V}}_{25}$ compared with the subjects given corn oil-rich supplementation. PEF values were increased in both two groups during the study. This was thought to be caused by the other therapies (drugs and respiratory rehabilitation) accompanying the diet therapy. During the improvement of the chronic obstructive pulmonary disease, the improvement of the PEF values tended to precede that of $\mathrm{FEV}_{1.0}(30$, 31 ). That might be the reason for the discrepancy in the PEF values and $\mathrm{FEV}_{1.0}$ values.

Several reports have failed to demonstrate a beneficial effect of EPA in patients with bronchial asthma $(32,33)$. This study revealed the efficacy of the perilla seed oil on asthma. Perilla seed oil is metabolized to EPA. Therefore, the action of perilla seed oil might include the effects of perilla seed oil itself in addition to the action of EPA.

We did not examine the change of serum IgE value and $n-3$ fatty acid/ n-6 fatty acid ratio during this study. Some researchers documented significant influences of dietary PUFA on the serum IgE and n-3 fatty acids/ n- 6 fatty acids ratio $(34,35)$.

The present study suggests that dietary supplementation with perilla seed oil, rich in $\alpha$-LNA, is more significantly beneficial to bronchial asthma than that with corn oil, rich in LA, by the suppressive effects on the generation of LTs by leucocytes.

The present study may be important to develop a diet therapy for asthmatic patients. Further studies are needed to develop a nutritionally balanced diet therapy for bronchial asthma.

\section{References}

1) Wenzel SE, Westcott JY, Larsen GL. Bronchoalveolar lavage fluid mediator levels $5 \mathrm{~min}$ after allergen challenge in atopic subjects with asthma: Relationship to the development of late asthmatic responces. J Allergy Clin Immunol 87: 540-548, 1991.

2) Kirby JG, Hargreave FG, Gleich GJ, O'Byrne PM. Bronchoalveolar cell profiles of asthmatic and nonasthmatic subjects. Am Rev Respir Dis 136: 379-383, 1987.

3) Kelly C, Ward C, Stenton CS, Hendrick DJ, Walters EH. Number and activitty of inflammatory cells in bronchoalveolar lavage fluid in asthma and their relation to airway responsiveness. Thorax 43: 684-692, 1988.

4) Pauwels R. The relationship between airway inflammation and hyperresponsiveness. Clin Exp Allergy 866: 207-215, 1983.

5) Thien FC, Walters EH. Eicosanoids and asthma: An update. Prostaglandins Leukot.Essent. Fatty Acids 52: 271-288, 1995.

6) Ashida K, Mitsunobu F, Mifune T, et al. A pilot study: Effects of dietary supplemention with $\alpha$-linolenic acid-enriched perilla seed oil on bronchial asthma. Allergol Intern 46: 181-185, 1997.

7) National Heart, Lung, and Blood Institute, National Institutes of Health. Bethesda, Maryland 20892. International consensus report on diagnosis and management of asthma. Eur Respir J 5: 601-641, 1992.

8) Tanizaki Y, Kitani H, Okazaki M, Mifune T, Mitsunobu F, Kimura I. Histamine and leukotriene $\mathrm{C} 4$ levels in bronchoalveolar lavage (BAL) fluid in patients with bronchial asthma. Jpn J Clin Immunol 116: 99-108, 1993.

9) Tanizaki Y, Kitani H, Okazaki M, Mifune T, Mitsunobu F, Kimura I. Changes in the proportions of bronchoalveolar lymphocytes, neutrophils and basophilic cells and the release of histamine and leukotrienes from bronchoalveolar cells in patients with steroid dependent intractable asthma. Int Arch Allergy Immunol 101: 196-202, 1993.

10) Jemionek JF, Contreras YJ, French JE, Shields LT. Technique for increased granulocyte recovery from human whole blood by counterflow centrifugation elutriation. 1. In vitro analyses. Transfusion 19: 120-128, 1979.

11) Tanizaki Y, Sudo M, Kitani H, et al. Release of heparin-like substance and histamine from basophilic leucocytes separated by counterflow centrifugation elutriation. Jpn J Med 29: 356-361, 1990.

12) Lam S, Chan H, LeRiche JC, Chan-Yeung M, Salari H. Release of 


\section{Effects of n-3 Fatty Acids on Asthma}

leukotrienes in patients with bronchial asthma. J Allergy Clin Immunol 81: 711-717, 1988.

13) Lee TH, Mencia-Huerta JM, Shih C, Corey EJ, Lewis RA, Austen KF. Effects of exogenous arachidonic, eicosapentaenoic, and docosahexaenoic acids on the generation of 5-lipoxygenase pathway products by ionophoreactivated human neutrophils. J Clin Invest 75: 1922-1933, 1984.

14) Prescott SM. The effect of eicosapentaenoic acid on leukotriene B production by human neutrophils. J Biol Chem 259: 7615-7621, 1984.

15) Taniguchi $Y$, Tamura G, Honma $M$, et al. The effect of an oral leukotriene antagonist, ONO-1078, on allergen-induced immediate bronchoconstriction in asthmatic subjects. J Allergy Clin Immunol 92 (4): 507-512, 1993.

16) Hamilton AL, Watson RM, Wyile G, O’Byrne PM. Attenuation of early and late phase allergen-induced broncho-constriction in asthmatic subjects by a 5-lipoxygenase activating protein antagonist, BAYX1005. Thorax 52: 348-354, 1997.

17) von Schacky C, Kiefl R, Jendraschak E, Kaminski WE. n-3 fatty acids and cysteinyl-leukotriene formation in humans in vitro, ex vivo, and in vivo. J Lab Clin Med 121: 302-309, 1993.

18) Broughton KS, Johnson CS, Pace BK, Liebman M, Kleppinger KM. Reduced asthma synptoms with n-3 fatty acid ingestion are related to 5series leukotriene production. Am J Clin Nutr 65: 1011-1017, 1997.

19) von Schacky C. Prophylaxis of atherosclerosis with marine omega-3 fatty acids-comprehensive strategy. Ann Intern Med 107: 890-899, 1987.

20) Belch JJ, Ansell D, Madhok R, O'Dowd A, Sturrock RD. Effects of altering dietary essential fatty acids on requirements for non-steroidal antiinflammatory drugs in patients with rheumatoid arthritis: a double blind placebo controlled study. Ann Rheum Dis 47: 96-104, 1988.

21) Cleland LG, French JK, Betts WH, Murphy GA, Elliott MJ. Clinical and biochemical effects of dietary fish oil supplements in rheumatoid arthritis. J Rheumatol 15: 1471-1475, 1988.

22) DiGiacomo RA, Kremer JM, Shah DM. Fish oil dietary supplementation in patients with Raynaud's phenomenon: a double blind, controlled, prospective study. Am J Med 86: 158-164, 1989.

23) van der Tempel H, Tulleken JE, Limburg PC, Muskiet FA, van Rijswijk MH. Effects of fish oil supplementation in rheumatoid arthritis. Ann Rheum Dis 49: 76-80, 1990.

24) Lorenz R, Weber PC, Szimnau P, Heldwein W, Strasser T, Loeschke K. Supplementation with $\mathrm{n}-3$ fatty acids from fish oil in chronic inflamma- tory bowel disease -a randomized placebo controlled, double-blind crossover trial. J Intern Med Suppl 225: 225-232, 1989.

25) Ziboh VA. Implications of dietary oils and polyunsaturated fatty acids in management of cutaneous disorders. Arch Dermatol 125: 241-245, 1989.

26) Arm JP, Horton CE, Mencia-Huerta JM, et al. Effect of dietary supplementation with fish oil on mild asthma. Thorax 43: 84-92, 1988.

27) Arm JP, Horton CE, Spur BW, Mencia-Huerta JM, Lee TH. The effects of dietary supplementation with fish oil lipids on the airways response to inhaled allergen in bronchial asthma. Am Rev Respir Dis 139: 13951400, 1989.

28) Dry J, Vincent D. Effect of a fish oil diet on asthma: Results of a 1 year double blind study. Int Arch Allergy Appl Immunol 95: 156-157, 1991.

29) Thien FC, Mencia-Huerta JM, Lee TH. Dietary fish oil effects on seasonal hay fever and asthma in pollen-sensitive subjects. Ann Rev Respir Dis 147: 1138-1143, 1993.

30) Mitsunobu F, Mifune T, Hosaki Y, et al. Effects of spa therapy on patients with pulmonary emphysema -relationship to disease severity evaluated by low attenuation area of the lung on high resolution computed tomography - The Journal of Japanese Association of Physical Medicine Balneology and Climatology 61: 79-86, 1998.

31) Mitsunobu F, Mifune T, Hosaki $Y$, et al. Improvement of pulmonary function by spa therapy in patients with emphysema, evaluated by residual volume (RV) and low attenuation area (LAA) of high-resolution computed tomography (HRCT). The Journal of Japanese Association of Physical Medicine Balneology and Climatology 62: 121-128, 1999.

32) Picado C, Castillo JA, Schinca N, et al. Effects of a fish oil enriched diet on aspirin intolerant asthmatic patients: a pilot study. Thorax 43: 93-97, 1988.

33) Stenius-Aarniala B, Aro A, Hakulinen A, Ahola I, Seppala E, Vapaatalo $H$. Evening primrose oil and fish oil are ineffective as supplementary treatment of bronchial asthma. Ann Allerg 62: 534-537, 1989.

34) Koga T, Nonaka M, Gu JY, Sugano M. Linoleic and alpha-linolenic acids differently modify the effects of elaidic acid on polyunsaturated fatty acid metabolism and some immune indices in rats. Br J Nutr 77: 645-656, 1997.

35) Watanabe S, Sakai N, Yasui Y, et al. A high alpha-linolenate diet suppresses antigen-induced immuno-globulin $\mathrm{E}$ response and anaphy-lactic shock in mice. J Nutr 124: 1566-1573, 1994. 